\title{
El diálogo transdisciplinario un enfoque de abordaje del cambio climático
}

\author{
Juan Ignacio Alfaro Mardones ${ }^{1}$ \\ Carmen de Jesús Fernández Hernández ${ }^{2}$ \\ Manuel de Jesús González García ${ }^{3}$
}

\section{RESUMEN}

La transdisciplinariedad es una forma de generación del conocimiento dialogada. Los saberes tradicionales de las ciencias naturales y de las ciencias sociales debaten abiertamente con la experiencia empírica acumulada en las personas y organizaciones sociales. Mediante este diálogo a universidad y las organizaciones campesinas buscan estrategias de adaptación al cambio climático que les permita mejorar sus condiciones de vida y augurar un futuro para sus familias. Este artículo describe una fase del diálogo transdisciplinar del II semestre del 2014, a partir de un mapeo de las parcelas y la construcción de las memorias comunitarias. El reconocimiento en la responsabilidad por la destrucción de la tierra y la reflexión sobre la vida de esa tierra permite pensar en estrategias para sobrevivir con la destrucción y cuidar esa vida. La identificación en cada comunidad de los niveles de vulnerabilidad y de resiliencia de cada parcela abre la puerta a planes de finca que enfoquen la transformación productiva a una estrategia familiar y organizacional de desarrollo humano y adaptación al cambio climático.

Palabras clave: Transdisciplinariedad, investigación acción participativa, adaptación al cambio climático, resiliencia y desarrollo.

Recibido: 18 de marzo de 2015

Aceptado: 15 de junio de 2015

1 Docente investigador FAREM Matagalpa. Estudiante de doctorado en desarrollo rural territorial sustentable. Correo Electrónico: altarrib@ibw.com.ni

2 Docente investigadora FAREM Matagalpa. Estudiante de doctorado en desarrollo rural territorial sustentable. Correo Electrónico: carmenferher@yahoo.com

3 Docente investigador FAREM Matagalpa. Estudiante de doctorado en desarrollo rural territorial Correo

Electrónico: sustentable.mjesus14@yahoo.com 


\title{
Transdisciplinary dialogue as an approach to study climate change
}

\begin{abstract}
Transdisciplinarity is a form of knowledge generation dialogue. The traditional knowledge of the natural and social sciences are openly debated with accumulated empirical experience in people and social organizations. Through this dialogue to college and peasant organizations, they seek adaptation strategies to climate change that would enable them to improve their living conditions and predict a future for their families. This article describes a transdisciplinary dialogue in the second semester of 2014, from a mapping of plots and construction of community memories. The recognition of responsibility for the destruction of the earth and reflection on the life of that land allow thinking of strategies to survive the destruction and take care of that life. The identification in each community about the levels of vulnerability and resilience of each plot opens up farm plans that focus on productive transformation to a family and organizational strategy of human development and climate change adaptation.
\end{abstract}

Keywords: Transdisciplinarity, participatory action research, climate change adaptation, resilience and development. 


\section{INTRODUCCIÓN}

La Universidad Nacional Autónoma de Nicaragua, mediante su Facultad Regional Multidisciplinaria de Matagalpa (UNAN-FAREM-Matagalpa) ha establecido alianzas con la Unión de Campesinos Organizados de San Dionisio (UCOSD) a partir de una serie de acciones concretas en diferentes marcos de actuación: tesis de grado y prácticas profesionales desde las carreras de ingeniería agronómica y economía; tesis de maestría en el programa de Maestría en desarrollo rural territorial sustentable; una propuesta de investigación, en el marco del proyecto de la Sociedad Rural, Economía y Recursos Naturales, Integrando competencias en el Desarrollo Rural" (SERIDAR) (Sociedad rural, economía y recursos naturales. Integrando competencias en el desarrollo rural. , 2013); y actualmente tres tesis en el programa de Doctorado en Desarrollo rural territorial sustentable que tratan de articular las prácticas y las tesis de investigación de pregrado de la carrera de economía con este programa de postgrado.

De este vínculo, surgió la demanda de la organización campesina para que la universidad les acompañara mediante un proceso participativo y transdisciplinario, en el análisis de la realidad socioeconómica y productiva de las familias y comunidades, que permitiera generar un conjunto de propuestas de desarrollo y de adaptación al cambio climático.

¿Cómo está influyendo la asociatividad entre campesinos para generar capacidad de resiliencia y adaptación al cambio climático? ¿Quiénes son los agricultores del futuro en la subcuenca del Río Cálico? Una vez identificadas estas primeras preguntas vinculadas al desarrollo interno de las personas asociadas a esta organización campesina, se comenzó un proceso de reflexión comunitaria realizado en el año 2013 en el marco del proyecto SERIDAR. Desde enero del 2014 hasta junio 2014 se acompañó un proceso de reflexión comunitaria, que permitió cruzar el plan organizativo institucional de la UCOSD con las carencias y las potencialidades desde cada una de las comunidades. Esta información fue analizada conjuntamente con el consejo directivo de la UCOSD y el equipo transdisciplinar de la FAREM Matagalpa para definir las acciones de investigación que determinaría la continuidad de este diálogo entre la academia y el campesinado en el territorio de San Dionisio. En este artículo se analiza el diálogo realizado en el II semestre del 2014 continuidad de las fases anteriormente descritas.

\section{MATERIALES Y MÉTODOS}

\section{La Investigación acción participativa como marco metodológico para el diálogo transdisciplinario.}

"Para iniciar, es preciso recordar que entre 1960 y 1970 se fue gestando en América Latina una corriente amplia de pensamiento en la que confluyeron la Educación Popular, la Teología de la Liberación, la Comunicación Alternativa, la Investigación Acción Participativa y la Filosofia de la Liberación (Torres, 2007). Esta corriente de pensamiento estaba orientada por lo que hoy se conoce como el "paradigma emancipatorio." (Ortiz \& Borjas, 2008) La Unión de Campesinos Organizados de San Dionisio (UCOSD), es un sujeto colectivo que ha buscado el desarrollo de sus asociados. A partir de un proceso de reflexión colectiva, iniciado hace aproximadamente hace treinta años. La universidad está desarrollando un dialogo con esta institución y sus participantes, para contrastar sus conocimientos adquiridos en este tiempo, con el saber teórico acumulado por las investigaciones de los docentes involucrados en esta alianza de investigación. Un posicionamiento metodológico con la Investigación acción participativa (IAP), "procesos de investigación en un camino en "espiral” según palabras del educador australiano Stephen Kemmis (1989), quien encontró en esta modalidad de investigación el fundamento de una ciencia social crítica bien demarcada de las corrientes positivistas y hermenéuticas" (Ortiz \& 
Borjas, 2008). La necesidad de usar una metodología que se desmarque del reduccionismo o fragmentación que se ha caído, tanto desde el método positivista de las ciencias naturales, como en la hermenéutica cerrada de las ciencias sociales. Sobre todo un método que nos permita comprometernos con los efectos del cambio climático "Deberá apelarse a los intereses a largo plazo en formas desusadas. La vida de los hijos y nietos. En efecto, va más allá todavía, depende del amor a la tierra que los seres humanos sentían intensamente antes, pero que se ha desvanecido y deteriorado. (...) No ha muerto totalmente, en el corazón humano, el amor por la tierra. Todavía hay cierta preocupación por que los hijos y los nietos hereden un mundo habitable." (Daly \& Cobb, 1989)

La investigación se realiza inspirada en la Investigación Acción Participativa, como un método que facilita la transformación social, al mismo tiempo que la generación del conocimiento Es una investigación con enfoques cuantitativos en diálogo permanente con enfoques cualitativos y con la aplicación del método teórico, a partir de una reflexión sobre teorías del desarrollo y el empírico, para tratar de hacer dialogar estas propuestas teóricas con la realidad y el saber local, desde un diálogo transdisciplinario, tanto con las personas protagonistas de la UCOSD, así como con el equipo investigador de la UNAN-Managua. "la tensión entre teoría y práctica que conduce a un diálogo entre saberes teóricos y saberes prácticos convirtiendo al investigador en un educador desde el principio freiriano de la "concientización dialógica". (Ortiz \& Borjas, 2008). El carácter etnográfico de la investigación parte de la convivencia del investigador con los y las protagonistas del desarrollo rural dentro de los territorios en el transcurso de la acción dialógica. Desde el 2013 se han realizado más de dos sesiones de reflexión comunitaria en cada una de las 13 comunidades donde tiene influencia la organización y se lleva un diálogo permanente con el consejo directivo de la organización. "El conocimiento avanza entonces como una espiral en que se procede de lo más sencillo a lo más complejo, de lo conocido a lo desconocido, todo en contacto permanente con las bases y los grupos "ad hoc" de referencia. De éstos se reciben los datos; se actúa con ellos; se digiere la información en un primer nivel; y se reflexiona a un nivel más general. Luego se devuelven los datos de manera más madura y ordenada; se estudian los efectos de esta devolución y asi indefinidamente, aunque dentro de plazos prudenciales determinados por la lucha misma y sus necesidades" (Fals Borda, 1987).

\section{El mapeo agrario de parcelas y la memoria comunitaria como herramientas de diálogo.}

La información obtenida con la matriz de necesidades y satisfactores se presentó de forma transdisciplinaria con el Consejo directivo de la UCOSD y se cruzó con las líneas y acciones priorizadas en su plan estratégico. De este debate que llevó desde mayo hasta agosto del 2014, se pudieron identificar los siguientes aspectos centrales que se pusieron en el centro del esfuerzo investigativo $\mathrm{y}$ de transformación: 1. Aspectos organizativos y de desarrollo humano. 2. Aspectos productivos y de gestión empresarial y 3. Aspectos ambientales y de adaptación al cambio climático. Al mismo tiempo se identificaron los aspectos (indicadores) que se querían abordar en cada una de las asambleas comunitarias. (Imágenes 1, 2 y 3).

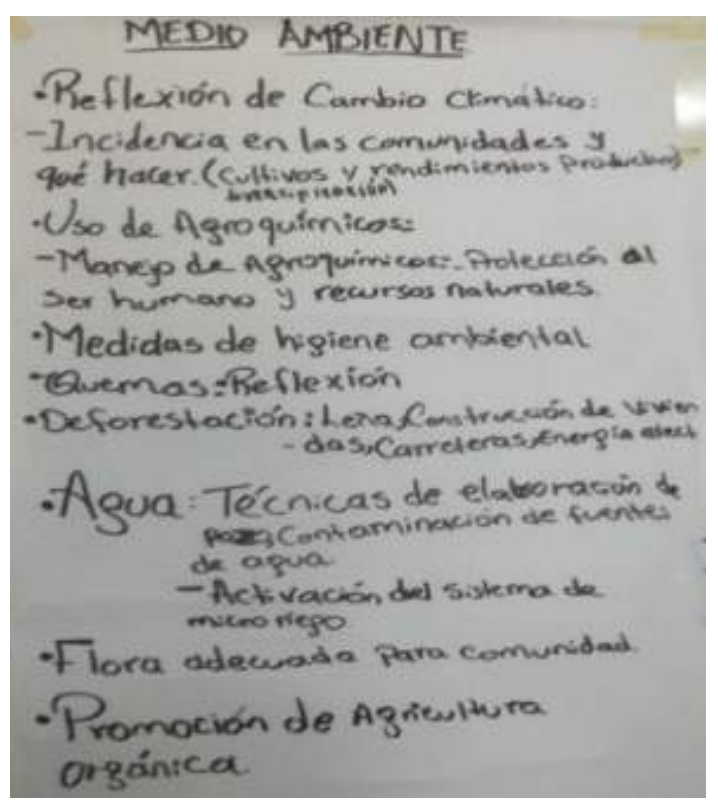




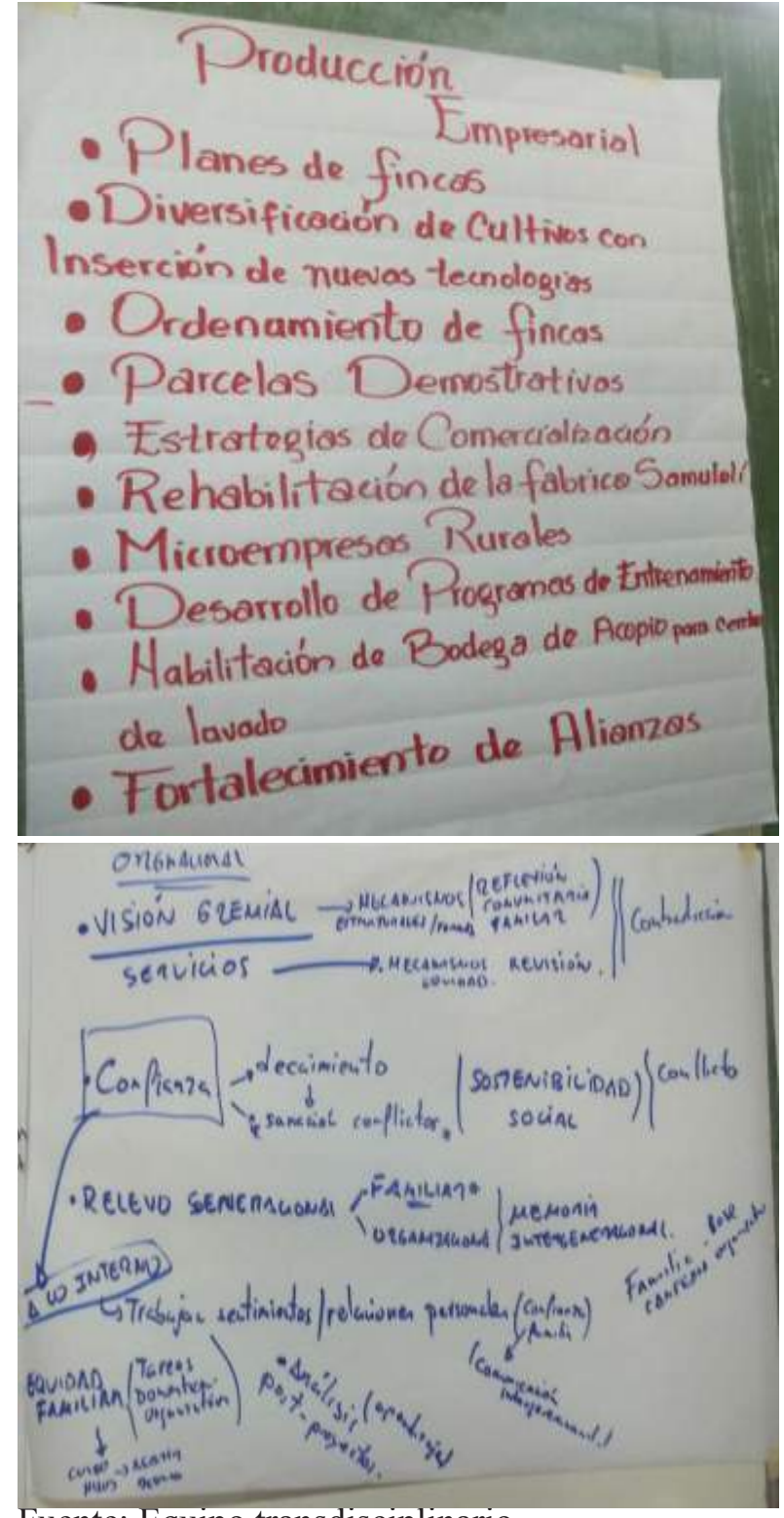

Fuente: Equipo transdisciplinario.

\section{Cuadro 1. Operacionalización de variables}

\begin{tabular}{|l|l|l|}
\hline \multicolumn{3}{|c|}{ Operacionalización de variables } \\
\hline Variables & Sub-variables & \multicolumn{1}{|c|}{ Indicadores } \\
\hline \multirow{4}{*}{ Ambiental } & \multirow{2}{*}{ Biosfera } & Bosque \\
\cline { 3 - 3 } & Agua \\
\cline { 3 - 3 } & $\begin{array}{l}\text { Sistema de } \\
\text { producción }\end{array}$ & Suelo \\
\cline { 3 - 3 } & & Fertilizante \\
\cline { 3 - 3 } & & Semilla \\
\hline
\end{tabular}

\begin{tabular}{|c|c|c|}
\hline \multirow{6}{*}{ Social } & \multirow{3}{*}{ Ingreso } & Ingreso/Fuente \\
\hline & & Ingreso/Cultivo \\
\hline & & Autoconsumo \\
\hline & \multirow{3}{*}{ Familiar } & Vivienda \\
\hline & & Energía \\
\hline & & $\begin{array}{l}\text { Estructura } \\
\text { familiar }\end{array}$ \\
\hline \multirow{6}{*}{ Económico } & \multirow{3}{*}{ Producto } & Área cultivo \\
\hline & & Rendimiento \\
\hline & & Ganado/aves \\
\hline & \multirow{3}{*}{ Empresarial } & Infraestructura \\
\hline & & Acopio \\
\hline & & $\begin{array}{l}\text { Canales de } \\
\text { comercialización }\end{array}$ \\
\hline
\end{tabular}

Fuente: Equipo transdisciplinar.

En dos sesiones de trabajo se discutió una metodología para llevar a las comunidades y poder dialogar con las familias y desde sus propias parcelas y se eligieron dos técnicas.

- Mapeo agrario (apoyado con un cuestionario para trabajar en familia)

- Memoria comunitaria apoyada en una línea del tiempo construyendo la historia comunitaria en su relación con la UCOSD desde los fundadores hasta la actualidad.

Se decidió calendarizar para el II semestre del 2014 13 asambleas de reflexión comunitaria con mapeo de parcelas y construcción de memoria comunitaria. A partir de esta acción se abordaron en forma dialogada y asamblearia los aspectos organizacionales, empresariales y ambientales que habían sido consensuados con los dirigentes campesinos.

De estas asambleas nacieron gran cantidad de reflexiones, propuestas, críticas que deben ser analizadas y procesadas para construir la última etapa de la investigación. 


\section{Diagrama 1. Etapas aplicadas en las asambleas} comunitarias
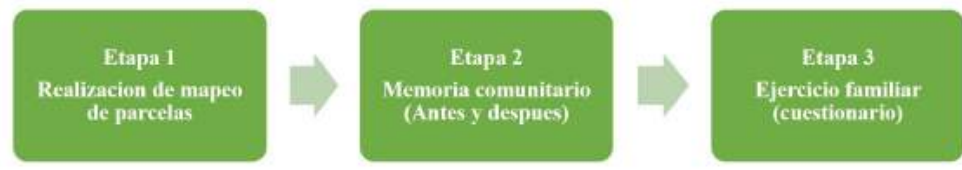

Fuente: Equipo transdisciplinar

La primera etapa consistió en el mapeo de la parcela familiar creado por ellos mismos en donde reflejarían el estado actual de los recursos. (diagrama 1 y 2 )

La segunda etapa consistió en un proceso de reflexión y análisis a través de una línea del tiempo donde, desde las personas fundadoras de la organización hasta llegar a las personas más recientemente asociadas, hacían un ejercicio crítico y reflexivo desde su memoria personal para reconstruir los aspectos que han funcionado, los que no han funcionado, los errores, aciertos $\mathrm{y}$ aprendizajes que han tenido como comunidad y la relación de la UCOSD con esta memoria colectiva.
Las memorias comunitarias se realizaron a partir de adecuar al territorio y las comunidades rurales la herramienta del perfil histórico de cada comunidad. "Las cronologías y tendencias históricas sirven para hacer visible de forma sencilla los acontecimientos históricos claves y los cambios más relevantes percibidos por la población local... A menudo, estos hechos históricos y su vivencia influyen sobre las decisiones futuras de los grupos locales. Es importante que quienes venimos de fuera tengamos en cuenta estos conocimientos y experiencias para entender los procesos locales de decisión en relación a las actividades." (Barroso, 2015) Finalmente se complementó toda esta información con un cuestionario que servía como vehículo para llevar la reflexión de la asamblea comunitaria a cada familia y que la familia dialogara sobre esta problemática y al mismo tiempo regresara por medio de éste sus aportes.

\section{Diagrama 2. Elementos a incluir en dibujos de parcela}

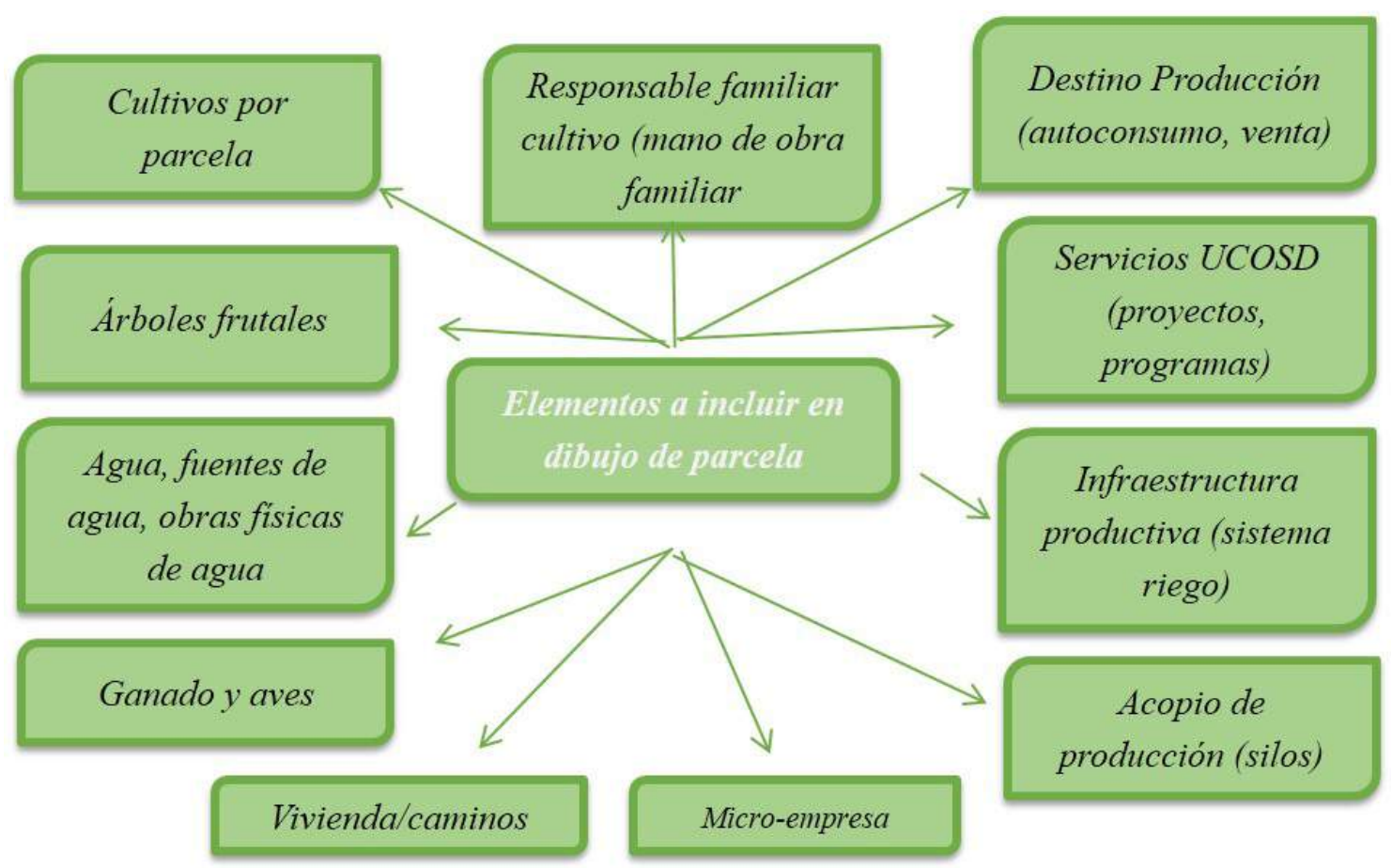

Fuente: Equipo investigador 
Una mirada personal desde cada parcela a la vulnerabilidad y resiliencia ante el cambio climático de las familias.

\section{Diagrama 3: Calificación de resiliencia}
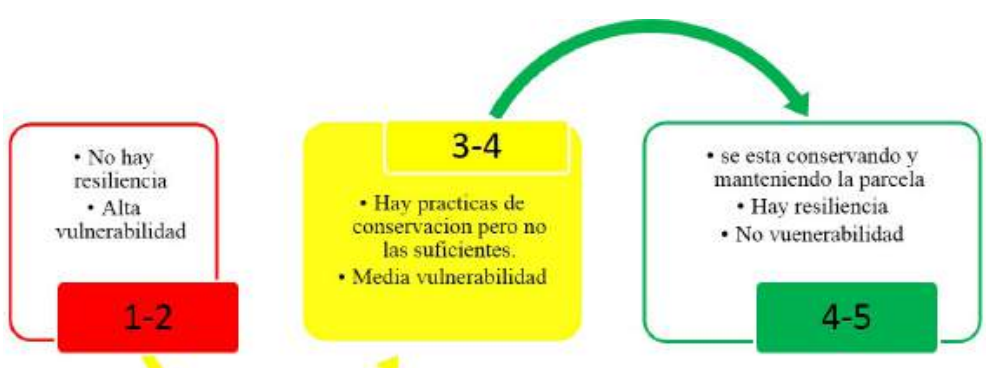

Fuente Adaptado de (Nicholls \& Altieri, 2013)
Mediante la información obtenida se creó una base de datos, utilizando como método de análisis la resiliencia socio- ecológica "propensidad de un sistema de retener su estructura organizacional y su productividad tras una perturbación" (Nicholls \& Altieri, 2013) es decir determinar la capacidad que tiene un sistema ante un evento climático, identificando si se están conservando y manteniendo las parcelas en las comunidades. Como parte de esta metodología de resiliencia se dio puntación a las parcelas, en base a criterios que se establecieron por variables e indicadores haciendo uso de los dibujos de las parcelas y los cuestionarios (cuadro 2). La existencia de resiliencia se determina de acuerdo a sus parámetros, calificación de 1 a 5. (Ver diagrama 3)

\section{Cuadro 2. Criterios de evaluación.}

\begin{tabular}{|c|c|c|c|c|c|}
\hline \multicolumn{6}{|c|}{ Criterios utilzados para purtuacion de indiadores } \\
\hline indicador & Instrumento & Puntuacion & indicador & Instrumento & Puntuacion \\
\hline \multirow{3}{*}{ Bosque } & \multirow{3}{*}{$\begin{array}{l}\text { Dibujo parcela, } \\
\text { cue stionario }\end{array}$} & $5=$ bosque + manejo & \multirow{3}{*}{ Energia } & \multirow{3}{*}{ Cuestionario } & $5=$ autosuficiencia \\
\hline & & $3=$ B osque sin manejo & & & $\begin{array}{l}3=\text { dependencia } \\
\text { en el hogar }\end{array}$ \\
\hline & & $1=$ No bosque & & & $1=$ de pendiente \\
\hline \multirow[b]{3}{*}{ A gua } & \multirow{3}{*}{$\begin{array}{c}\text { Dibujo parcela, } \\
\text { cue stionario }\end{array}$} & $\begin{array}{l}5=\text { Permanente } \\
\text { (Natural + proye cto) }\end{array}$ & \multirow{3}{*}{$\begin{array}{c}\text { Estructura } \\
\text { familiar }\end{array}$} & \multirow{3}{*}{ Cuestionario } & $5=$ equilibrio genera \\
\hline & & $3=$ Solo agua potable & & & $3=$ si hay muchos $n$ \\
\hline & & $1=$ No potable, A carrea agua & & & $1=$ solo adultos $\mathrm{y}$ a1 \\
\hline \multirow[b]{2}{*}{ Suelo } & \multirow[b]{2}{*}{ Cuestioanario } & $5=$ algun manejo & \multirow{3}{*}{$\begin{array}{l}\text { Area de } \\
\text { cultivo }\end{array}$} & \multirow{3}{*}{$\begin{array}{l}\text { Dibujos } \\
\text { parcelas }\end{array}$} & $5=$ diversificado \\
\hline & & $1=$ ningun manejo & & & $\begin{array}{l}\text { 3= semidiversificad } \\
\text { (Maiz, Frijol, Millo) }\end{array}$ \\
\hline \multirow[b]{2}{*}{ Fertilizante } & \multirow{3}{*}{ Cuestioanario } & $5=$ quimico y organico & & & $\begin{array}{l}1=\text { no diversicado } \\
\text { menos de } 3 \text { cultivo }\end{array}$ \\
\hline & & $1=$ quimico & \multirow{2}{*}{ Rendimie nto } & \multirow{2}{*}{ Cuestionario } & $5=>$ que el promed \\
\hline \multirow{3}{*}{ Semilla } & & $5=$ criolla & & & $1=<$ que el promed \\
\hline & \multirow[t]{2}{*}{ Cuestioanario } & $3=$ acriolla da & \multirow{3}{*}{ Ganado/aves } & \multirow{3}{*}{$\begin{array}{l}\text { Dibujos } \\
\text { parcelas }\end{array}$} & $5=$ va cas, cerdos, $g$ \\
\hline & & $1=$ mejorada & & & $3=$ algunas aves de \\
\hline \multirow{3}{*}{ Control de plaga } & \multirow{3}{*}{ Cuestioanario } & 5 - biologico & & & $1-$ nada \\
\hline & & $5=$ organico & \multirow{3}{*}{$\begin{array}{c}\text { Infraestructura } \\
\text { productiva }\end{array}$} & \multirow{3}{*}{$\begin{array}{l}\text { Dibujos } \\
\text { parcelas }\end{array}$} & $5=$ obras fisicas \\
\hline & & $1=$ quimico & & & $\begin{array}{l}3=\text { solo malla aves } c \\
\text { corral y establos }\end{array}$ \\
\hline \multirow{3}{*}{ uentes de ingres } & \multirow{3}{*}{ Cuestioanario } & $5=$ diversificado & & & $1=$ nada \\
\hline & & $3=$ autoconsumo jornalero & \multirow{3}{*}{ A copio } & \multirow{3}{*}{$\begin{array}{l}\text { Dibujos } \\
\text { parcelas }\end{array}$} & $5=$ silos \\
\hline & & $1=$ solo autoconsumo & & & $3=$ barriles \\
\hline \multirow{3}{*}{$\begin{array}{l}\text { ingresos } \\
\text { por cultivo }\end{array}$} & \multirow{3}{*}{ Cuestioanario } & $\begin{array}{l}5=\text { a ma yor rubro } \\
\text { comercializado }(100 \%)\end{array}$ & & & $1=\mathrm{sacos}$ \\
\hline & & $\begin{array}{l}3=\text { rubros comercializados } \\
\text { (Maiz, Frijol, Millon) }\end{array}$ & \multirow{3}{*}{$\begin{array}{l}\text { Canales de } \\
\text { comercializaci } \\
\text { on }\end{array}$} & & $5=\mathrm{UCOSD}$ \\
\hline & & $\begin{array}{l}1=\text { menos rubros } \\
\text { comercializados }\end{array}$ & & Cuestionario & $3=$ mercado \\
\hline & & $\begin{array}{l}5=\text { mayor rubro que } \\
\text { consume }(100 \%)\end{array}$ & & & $1=$ intermediario \\
\hline A utoconsumo & Cuestioanario & $\begin{array}{l}3=\text { rubros comercializados } \\
\text { (Maiz, Frijol, Millon) }\end{array}$ & & & \\
\hline & & $1=$ menor rubro que consume & & & \\
\hline Vivienda & Cuestioanario & $\begin{array}{l}5=\text { adecua do } \\
\text { (ladrillo, madera, minifalda) }\end{array}$ & & & \\
\hline & & $1=$ ina decuado & & & \\
\hline
\end{tabular}

\section{RESULTADOS Y DISCUSIÓN}

\section{La necesidad de la transdisciplinariedad en la investigación}

"Vale la pena preguntarse si el cambio climático, la crisis energética, la alimentaria y la financiera, sumados al crecimiento del número de personas pobres, la pérdida de la biodiversidad, entreotros, no constituyen una acelerada marcha de los acontecimientos que muestran la obsolescencia del desarrollo. Y cabe preguntarse también en qué medida las universidades no son un espacio privilegiado para colaborar en la promoción de nuevas ideas." (Agostino, Junio 2009)

En este diálogo con la Universidad, Saúl Úbeda, fundador de la UCOSD y actualmente coordinador contratado por el consejo directivo, nos recuerda desde su experiencia a la academia del peligro de la fragmentación. "Mis mejores momentos eran cuando todos los productores éramos parte de la asociación. La institucionalidad (burocratización)

Fuente: Equipo investigador. 
nos dividió y la profesionalización nos fragmentó." (Úbeda, 2014) La multidimensionalidad del desarrollo nos obliga a dialogar con diferentes disciplinas, tanto de las ciencias naturales, como de las ciencias sociales, así que necesitamos conocer los fundamentos epistemológicos y metodológicos de cada una de estas disciplinas para poder usar los criterios de validez y el proceso de investigación válido para cada uno de los aspectos abordados. El mundo vivido por personas que conviven en las zonas rurales está dotado de significados y profundidades que difícilmente podríamos interpretar sino se apoya en un diálogo transdisciplinario con el resto de los actores.

\section{La transdisciplinariedad en el abordaje del cambio climático}

Hasta ahora gran parte de los esfuerzos por abordar la crisis ecológica manifiesta desde hace más de cuarenta años han sido por parte de las ciencias ambientales, dejando a las ciencias sociales mucho más responsables de lado. "En An inquiry into the Human Prospect (1974), el economista Robert Heilbroner reflexionaba acerca del significado de esta presión de la economía humana sobre la biosfera. Consideraba especialmente los traumas políticos y sociales que se afrontan cuando ya no sea posible el crecimiento económico." (Daly \& Cobb, 1989) Es la actividad económica y la economía la disciplina que podría explicar las causas del cambio climático. "Más allá de cierto punto, el crecimiento material de la economía sólo se puede obtener a expensas del agotamiento del capital natural y socavando los servicios proporcionados por las funciones de soporto vital (aire, agua y alimentos sanos) de las que todos dependemos" (Merkel, 2005) Ha sido el diálogo entre ciencias ambientales y sociales, la economía ecológica, la que ha aportado luz a este asunto. "Lo que estamos diciendo en realidad es que la economía tiene una escala apropiada en relación con el ecosistema. Entendemos aquí por escala el tamaño físico, o sea la población multiplicada por las tasas de uso per cápita de recursos. ¡A medida que la economía crece, se vuelve más grande! A veces pasamos por alto las cosas más obvias. El ecosistema, del que la economía es un subsistema, no crece. Obviamente, el mundo no es estático. Pero es igualmente obvio que el diámetro de la Tierra no se expande. El flujo solar y las tasas de rotación de los ciclos biogeoquímicos permanecen aproximadamente constantes. En consecuencia, la economía se vuelve más grande en relación con el ecosistema y tensa en medida cada vez mayor al sistema matriz." (Daly \& Cobb, 1989) Pensar sobre modelos de desarrollo con la experiencia vivida en el siglo XX obliga a tener en cuenta la convivencia entre especies como condiciones necesarias para la reproducción y supervivencia del modelo.

Toda la riqueza que los trópicos regalan a sus habitantes está todavía atrapada y orientada al modelo agroexportador basado en productos primarios, esto representa en términos de economía ecológica un flujo de materiales y energía que empobrece en términos de economía real a la región cada día más. Es necesario buscar tecnologías apropiadas para la transformación de este flujo energético en satisfactores asimilables por las propias especies habitantes en estas zonas templadas o su traslado para otras regiones en términos de intercambio complementario, siempre con la sustentabilidad como criterio económico que fundamente la actividad productiva. "Sin embargo, no puede mantenerse sin modificaciones el modelo tradicional de crecimiento agropecuario de carácter extensivo... Este modelo de crecimiento extensivo se ha traducido en un masivo proceso de deforestación y deterioro de las cuencas hidrográficas, sedimentación de los ríos, aumento de la escorrentía y pérdida de biodiversidad, en conjunto con la vulnerabilidad ambiental que padece la población campesina que ha sido orillada a habitar y producir en las laderas de los cerros y las orillas de los ríos. (Acevedo Vogl, 2013)

Pensar que las soluciones generadas desde las ciencias ambientales son sustentables en medio de un modelo que necesita el crecimiento económico basado en 
el uso de recursos para su reproducción es utópico en su acepción más negativa. "La verdadera utopía negativa es pensar que el modelo de desarrollo actual es viable, la acumulación capitalista basada en el crecimiento económico está llegando a su límite y no por su cuestionamiento teórico; sino porque la naturaleza nos obliga a parar el crecimiento. Pasado el pico de extracción del petróleo y sin haber podido transformar la matriz energética global, las proyecciones energéticas determinan una disminución de la producción mundial a partir del 2020" (GarcíaDuran, 2008)

Es decir, no es una crisis ecológica solamente, sino una crisis del modelo económico y político, esta crisis se manifiesta en el cambio climático y hace legítima nuestra preocupación por los únicos pulmones y raíces sanas de nuestra existencia situados en las zonas rurales, citar una vez más una posición racional sobre la necesidad de una visión integral, que recoja un diálogo entre diferentes ciencias y con los y las protagonistas. "Los ecofilósofos mantienen que la crisis ecológica es el resultado de que los seres humanos no se dan cuenta de que simplemente son parte de la biosfera mayor. Pero eso pone el cuadro exactamente al revés, y no explica cómo el individuo humano no es una parte de la biosfera. Más bien, una parte de un individuo humano compuesto es una parte de la biosfera, y la biosfera misma es parte de la noosfera. Por esa razón, se puede instaurar la represión; por esa razón, la noosfera puede disociarse de la biosfera; por esa razón, el envenenamiento de nuestras raices significa también la muerte de nuestras ramas. La crisis ecológica es una psiconeurosis colectiva de amplitud mundial, es decir, una negación, alineación y disociación de biosfera y noosfera. Una represión de nuestro cuerpo común" (Wilber, 2005)

Así que no se trata de regresar a ser parte de la naturaleza, sino a sentir toda la naturaleza en nuestro interior (agua, células, tallo reptiliano, sistema límbico paleo mamífero...). "Nos tumbamos con los planetas y estrellas, los lagos y los ríos, el plancton y los robles, las lagartijas y los pájaros, los conejos y los simios, y lo repetimos no sólo porque son nuestros vecinos en el universo, sino porque son componentes de nuestro propio ser, son literalmente nuestros huesos y nuestra sangre, nuestro tuétano y nuestras tripas, nuestros sentimiento y nuestros miedos" (Wilber, 2005) De esta forma si reconozco toda la naturaleza en mí mismo, reconoceré que el problema ambiental es un acto suicida, no tanto una cuestión de "salvemos el planeta".

Por ahora los debates climáticos están atrapados en la esfera de los problemas externos y las soluciones externas; como lograr a través de un diálogo transdisciplinar que los y las protagonistas del cambio climático nos transformemos. Hoy puede existir un debate tecnológico, pero el debate educativo y liberador está siendo postergado "Los principales problemas de Gaia y su mayor amenaza no son la polución, la industrialización, la sobreexplotación de la tierra, del suelo, la superpoblación, la capa de ozono o cualquier otra cosa ...El principal problema ecológico es la falta de comprensión mutua y de entendimiento. El problema no está en cómo demostrar con pruebas científicas que la vida tiene problemas. En general, las pruebas de que es así son simples y abrumadoras. Cualquier puede entender los datos, pero a la mayoría de la gente no les importan. En otras palabras, el problema real no es exterior, sino interior. No es un problema de la ciencia, sino de la conciencia y la ética." (Wilber, 2005). Al final de un sabroso diálogo transdisciplinar parece que la crisis es del ser humano. Si llevamos este diálogo a la subcuenca del río Cálico ¿Cuál es la mochila que heredan los y las jóvenes en el territorio de San Dionisio? ¿Cómo está siendo el proceso de educación ambiental en sus escuelas y en sus parcelas? ¿Qué influencia está teniendo la asociatividad en los niveles de conciencia de los y las productoras de la subcuenca del río Cálico? 


\section{Algunos resultados del diálogo FAREM - UCOSD}

Un primer resultado de este ejercicio ha sido reflexionar de forma crítica sobre la responsabilidad de la propia comunidad y de cada socio o socia en los procesos de desarrollo y su responsabilidad en el cambio climático. Otro resultado ha sido la construcción con los y las protagonistas de los niveles de vulnerabilidad y resilencia alcanzados desde las familias en las parcelas en cada comunidad y de la memoria comunitaria y el papel de la UCOSD en la historia comunitaria.
Existen resultados de investigación y al mismo tiempo acciones de transformación; al poder tener una visión desde los participantes de sus parcelas, del papel de la UCOSD, de las carencias, potencialidades, aspectos comunitarios que funcionaron y que no funcionaron y una autoevaluación del ejercicio de estar asociados y organizados.

\section{Cuadro 3. Errores en la UCOSD}
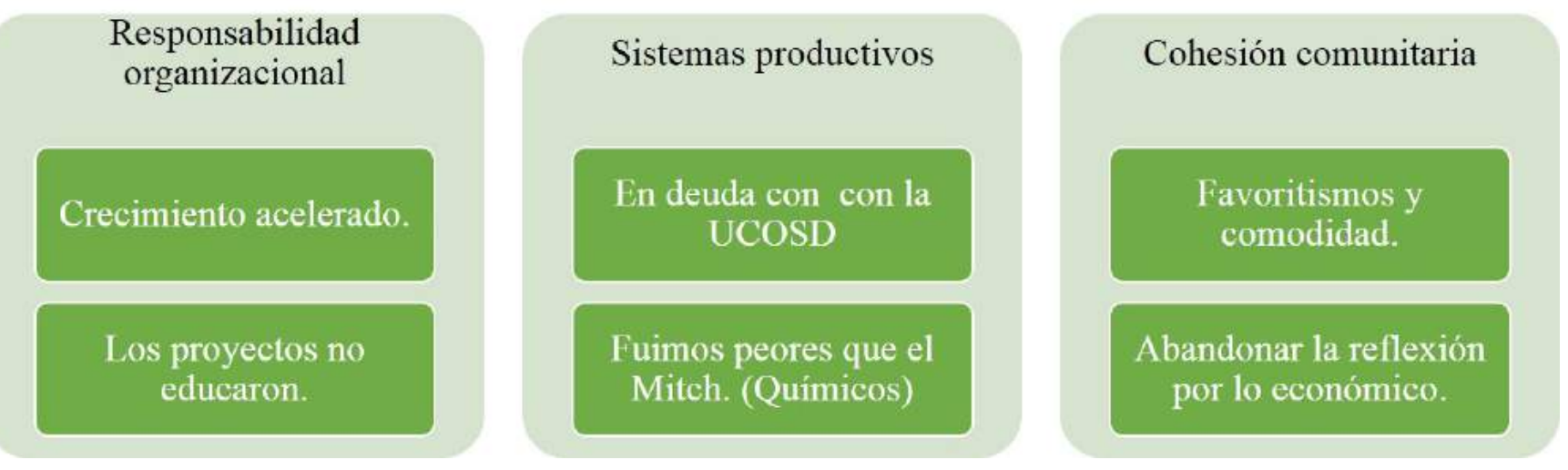

Fuente: Memorias comunitarias.

Los sistemas socio productivo y ambiental fueron maltratados por este mismo crecimiento, con acceso a deudas que transformaban la cultura laboriosa del machete en la cómoda cultura del químico. "Nosotros fuimos peores que el Mitch". Además del huracán Mitch que ha marcado un antes y un después en cada comunidad. Al mismo tiempo la UCOSD sirvió de espacio de sanación personal a personas que venían de años de conflictos armados, clandestinidad y luchas; era un lugar donde compartir y conocer a gente del "otro bando" y buscar intereses y motivaciones comunes. Un lugar que les ayudaba a tener y defender la tierra para pasar a ser dueños de su esfuerzo y de su vida. En definitiva, se creo una organización que después de 25 años se mantiene por que supo construir la respuesta a los problemas y donde permanecen todavía muchos de los que la construyeron.

\section{Cuadro 4. Éxitos de la UCOSD}

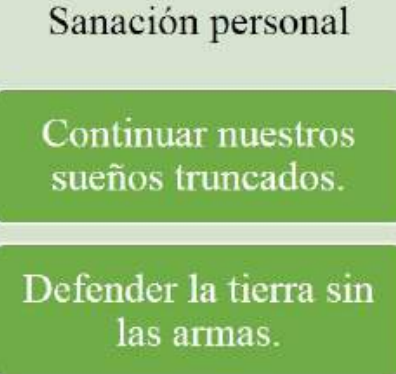

Fuente: Memorias comunitarias.
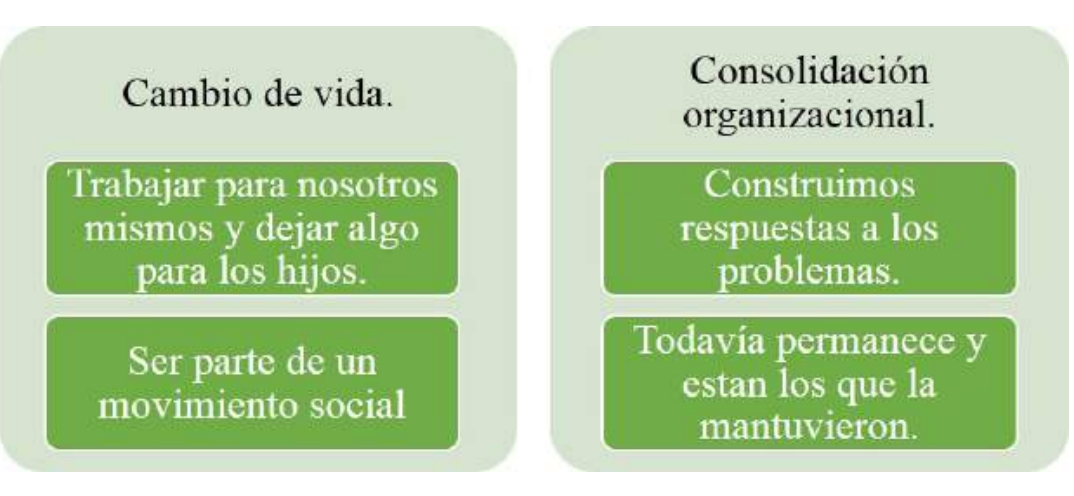
La construcción de la relación con la tierra, la defensa al acceso, la lucha por su mantenimiento en contradicción a su destrucción. Finalmente la UCOSD de hoy debe ser diferente a la UCOSD creada por los

\section{Cuadro 5. Aprendizajes desde la UCOSD}
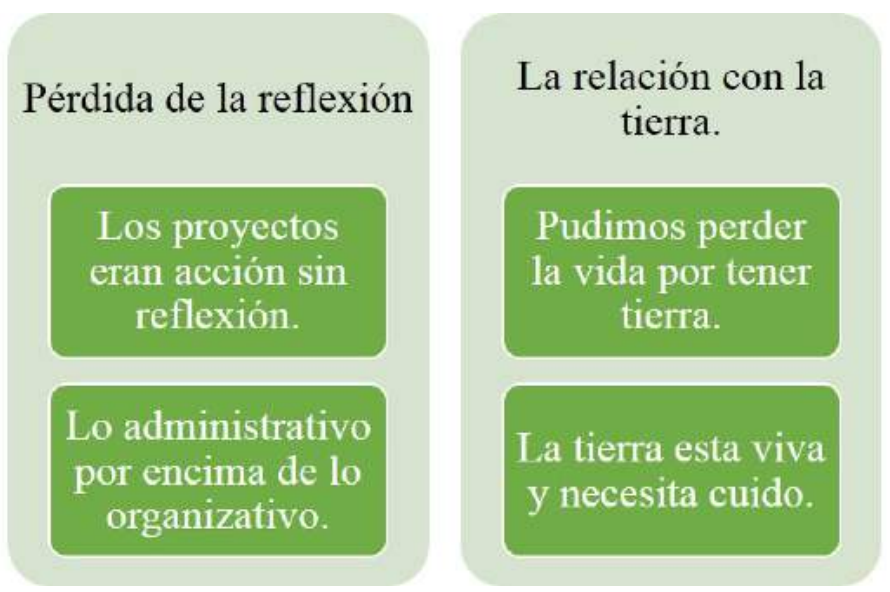

Fuente: Memorias comunitarias.

La UCOSD está viva en un territorio donde muchas organizaciones han nacido y muerto durante estos últimos veinte años. Empezó cuando no había otras organizaciones y persiste a pesar de sus muchos problemas. Ante este escenario se plantea como enfrentar una serie de realidades en su territorio: ¿Cómo convivir con la destrucción socio ambiental?, ¿cómo incluir a los que no son sujeto de servicios pero sí campesinos?, ¿cómo generar reservas alimentarias para construir mayor capacidad de resiliencia ante el cambio climático?, ¿cómo hacer que la tierra genere su propio pago sin maltratarla más?, ¿cómo trabajar una organización que complemente los valores de los fundadores con los de las nuevas generaciones?. fundadores, se necesitan equipos de trabajo en las comunidades que despierten la reflexión comunitaria para regresar el poder de la oficina administrativa hacia las comunidades.

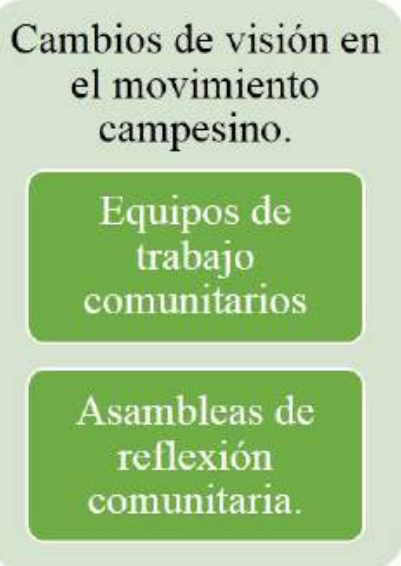

Cuadro 6. Interrogantes organizacionales.

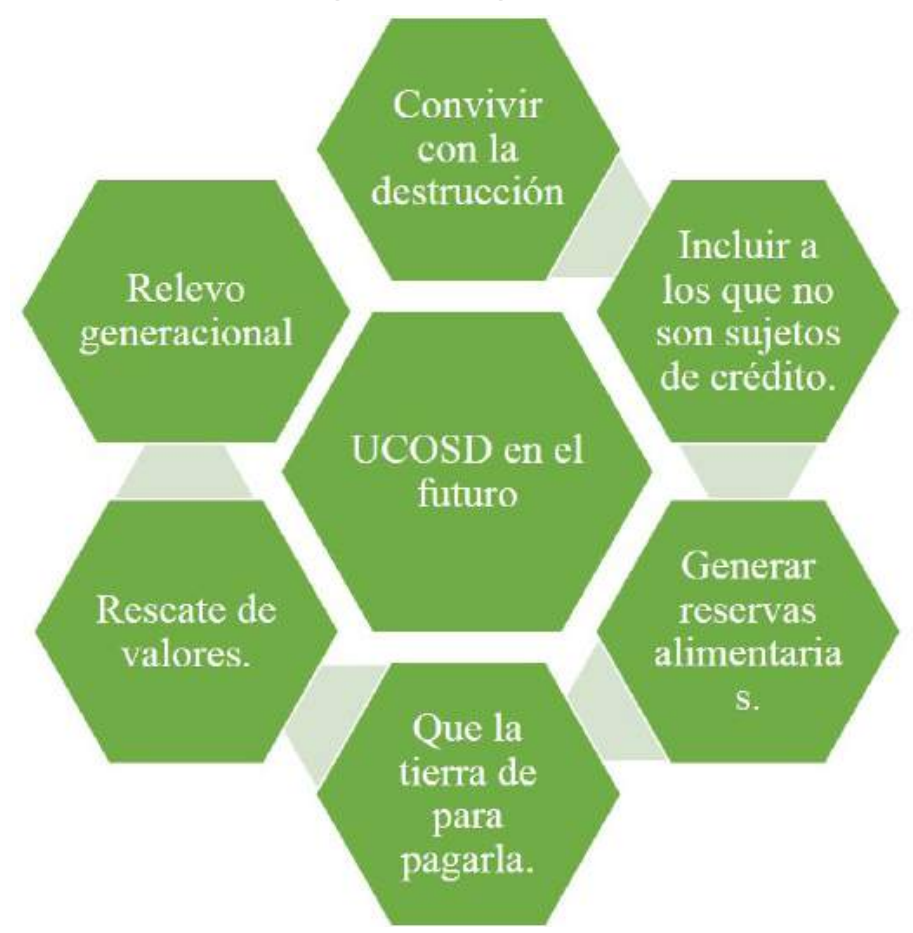

Fuente: Memorias comunitarias. 
Estado de la resiliencia y la vulnerabilidad de las comunidades.

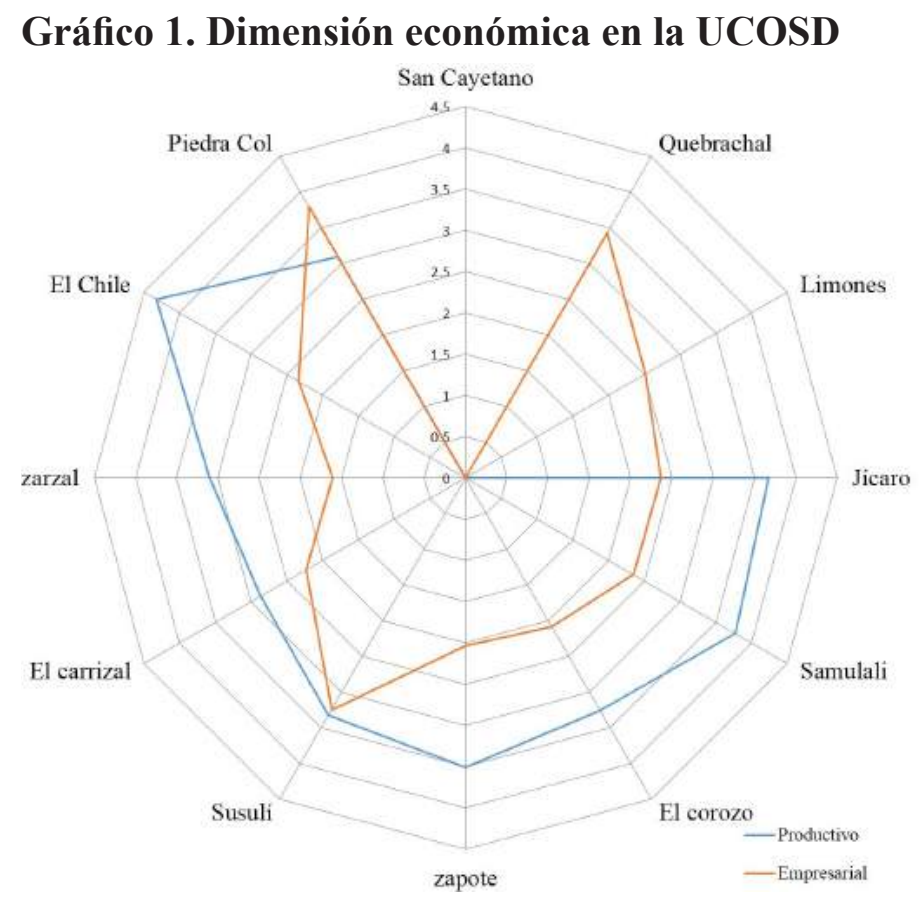

Fuente: Equipo investigador a partir del mapeo de parcelas.

Los niveles de resiliencia económica en la mayoría de las comunidades son medio y bajo, una crisis productiva y casi nulas iniciativas empresariales. Algunas de los proyectos se manifiestan en pequeña escala en torno a la posibilidad de acceso a agua.

\section{Gráfico 2. Dimensión ambiental en la UCOSD}

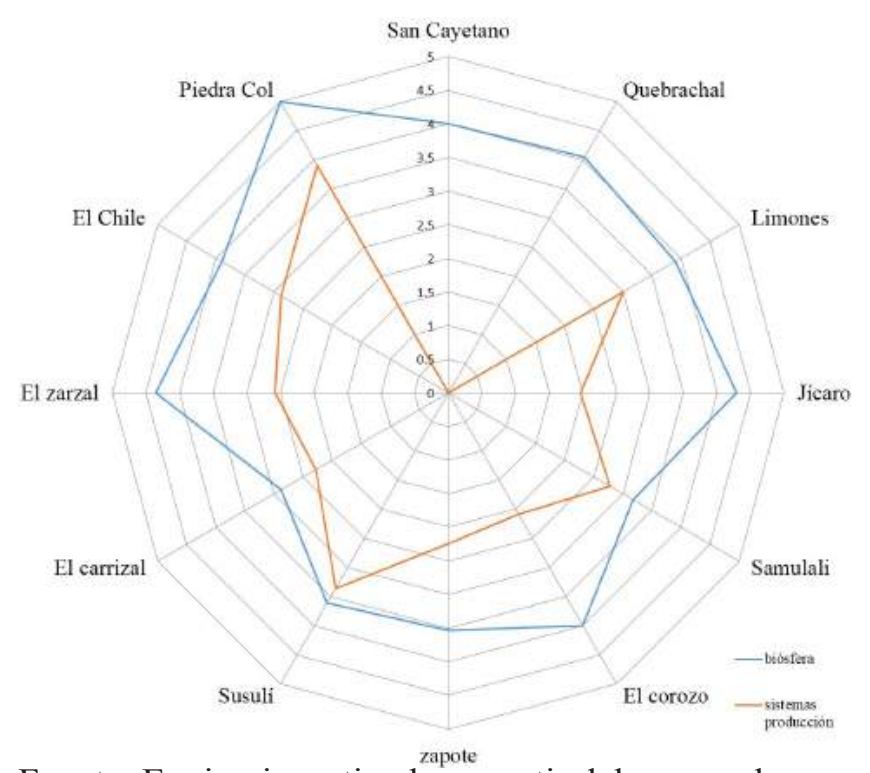

Fuente: Equipo investigador a partir del mapeo de parcelas.
En la dimensión ambiental hay diferencias entre el estado de la biósfera que todavía mantiene riqueza a pesar de la degradación del suelo y los sistemas productivos que son nocivos para el desarrollo ambiental. La vulnerabilidad del sistema ambiental y su capacidad de adaptación ante el cambio climático es una tarea pendiente en cada una de las comunidades. Los planes de finca deben apuntar a una reconversión socioproductiva que tenga el cuido ambiental como base de su elaboración.

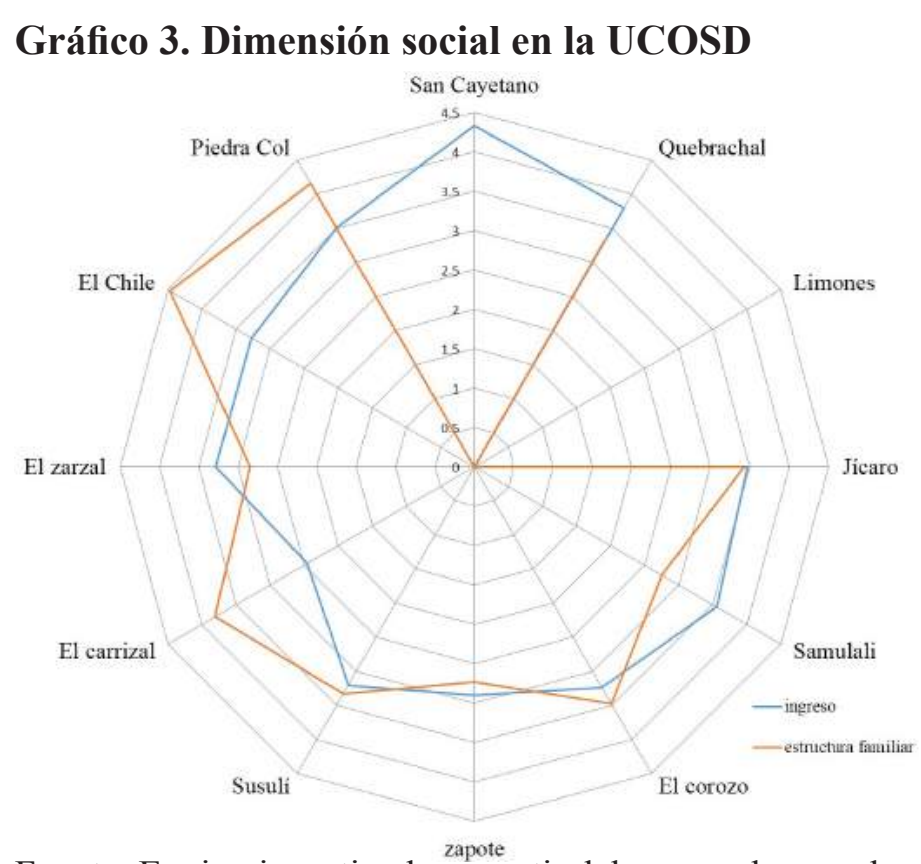

Fuente: Equipo investigador a partir del mapeo de parcelas.

En la dimensión social a pesar de mantenerse a niveles de resiliencia media, se constatan mejores perspectivas que en la dimensión económica, el acceso a la tierra, el banco de grano para el consumo, los cultivos de autoconsumo y la autonomía energética permiten tener mayor capacidad de adaptación ante cambios y crear estrategias de sobrevivencia que permitan afrontar transformaciones en las parcelas a medio plazo. 


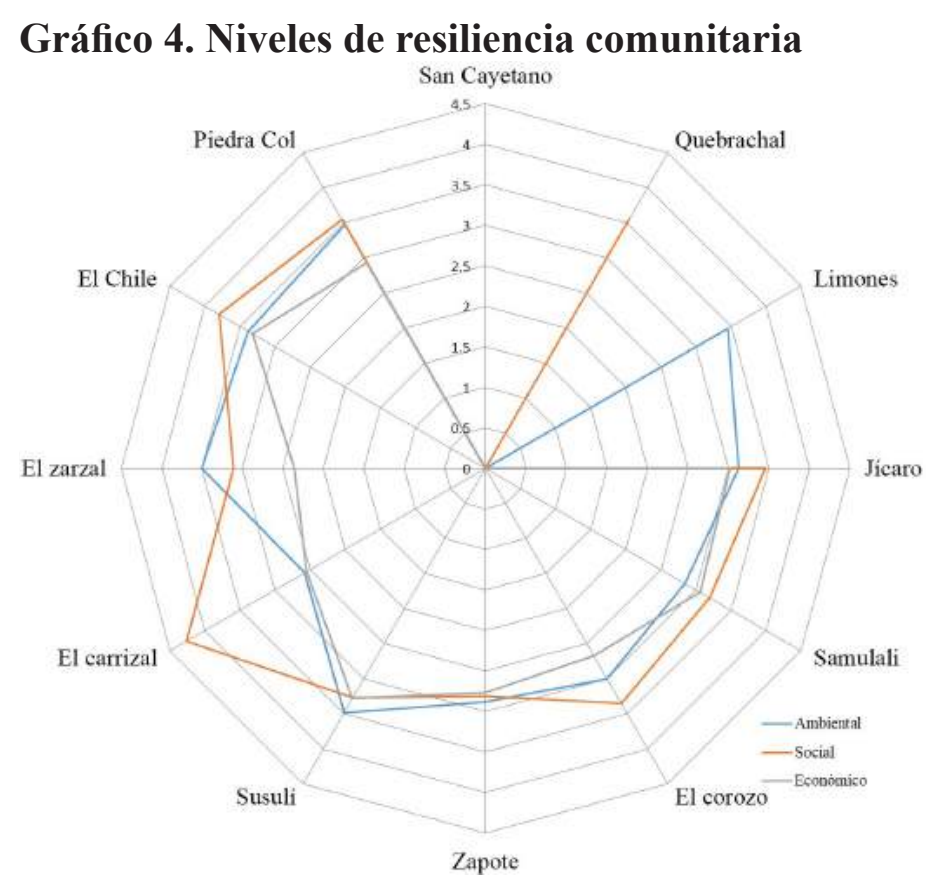

Fuente: Equipo investigador a partir del mapeo de parcelas.

\section{CONCLUSIONES}

Comunidades como el Jícaro, Samulalí, El Corozo, El Zapote, San Cayetano y Susulí mantienen un mayor equilibrio entre las tres dimensiones, pero en la mayoría la dimensión económica está mucho más vulnerable que la dimensión social. En la parte ambiental las situaciones difieren desde comunidades donde todavía se mantiene un equilibrio con el ecosistema como el Chile, El Quebrachal o Piedra Colorada y comunidades donde el deterioro es cada vez más grande como el Carrizal y Los limones. Desde una mirada global organizativa, esta organización tiene la mayor vulnerabilidad de sus asociados en la dimensión económica, existe una vulnerabilidad también grande en el plano ambiental, la mayor crisis de la subcuenca del río Cálico se manifiesta en el plano productivo y ambiental y en menor escala pero también con una vulnerabilidad media en el ámbito social pero con mayor margen ante los impactos climáticos y la globalización dependiente que viven las zonas rurales.

Este diálogo construyendo este nuevo conocimiento emergente continúa, cada comunidad debe tomar medidas de adaptación algunas comunitarias, otras desde las parcelas, en este contexto la organización campesina UCOSD debe cambiar su papel, sus funciones para adaptarlas a los cambios que la realidad le impone. La información está en manos del consejo directivo, pero también de cada familia, ahora se necesita crear la estrategia de desarrollo humano que les permita adaptarse al cambio climático.

¿Cómo el consejo directivo de la UCOSD acompañará este proceso? ¿Qué responsabilidad es asumirá a nivel familiar y comunitario? Al mismo tiempo la Universidad también requiere plantearse el modo en que va a continuar este diálogo. ¿Cómo voy a usar las prácticas y las investigaciones de pregrado para alimentar esta alianza? ¿Desde qué carreras y disciplinas quiero aportar a este diálogo? De alguna forma tanto desde la UCOSD como la FAREM Matagalpa se está experimentando con una nueva forma de construcción del conocimiento que podría ser un aporte metodológico a los esfuerzos que se realizan desde las personas e instituciones que aspiran a contribuir a la sobrevivencia de nuestra especie.

\section{BIBLIOGRAFÍA}

Acevedo Vogl, A. (19 demarzo de 2013). Características del desarrollo y del subdesarrollo. La Prensa, pág. $3 C$.

Agostino, A. (Junio 2009). Alternativas al desarrollo en América Latina: ¿Qué pueden aportar las Universidades? Revista de ALAI Sede Ecuador, 14-17.

Alfaro Mardones, J. I., Fernádez Hernádez, C., \& González García, M. (2014). Investigación acción participativa UCOSD - FAREM Matagalpa. Susuli: UCOSD - FAREM Matagalpa.

Alfaro, J., Fernandez, C., Calvo, E., \& González, M. (2014). La transdisciplinariedad en el desarrollo rural. Un estudio de caso: La Unión de Campesinos Organizados de San Dionisio (UCOSD), Matagalpa. Matagalpa. 
Barroso, C. (marzo de 2015). Los instrumentos de la investigación acción participativa. Recuperado el 10 de marzo de 2015, de www.ull.es: http:// www.ctinobar.webs.ull.es/1docencia/Cambio\%20 Social/

Daly, H., \& Cobb, J. (1989). Para el bien común. Reorientando la economía hacia la comunidad, el ambiente y un futuro sostenible. México: Fondo de cultura económico.

Fals Borda, O. (1987). Ciencia propia y colonialismo intelectual. Los nuevos rumbos. Bogotá: Carlos Valencia editores.

García-Duran, R. (2008). Contra la miseria de la economía, hacia la plenitud de la fraternidad. Barcelona: Ediciones Attac Catalunya.

Mardones, J. M., \& Ursua, N. (1999). Filosofía de la Ciencias Humanas y Sociales. México: Ediciones Coyoacán.

Martínez Miguélez, M. (1999). La nueva ciencia: su desafio, lógica y método. México: Trillas.

Max-Neef, M., Elizalde, A., \& Hopenhayn, M. (1986). Desarrollo a escala humana: una opción para el futuro. Santiago de Chile: Centro de alternativas de desarrollo.
Merkel, J. (2005). Simplicidad radical. Barcelona: Fundación Ferrer y Guardia.

Nicholls, C., \& Altieri, M. (2013). Agroecología y cambio climático. REDAGRES.

Ortiz, M., \& Borjas, B. (2008). La Investigación Acción Participativa: aporte de Fals Borda a la educación popular. Espacio Abierto, Vol. 17, Núm. 4, 615-627.

Sociedad rural, economía y recursos naturales. Integrando competencias en el desarrollo rural. . (2013). Proyecto de actualización de los enfoques y estrategias de desarrollo de la UCOSD. Matagalpa: SERIDAR - UNAN FAREM Matagalpa.

Úbeda, S. (11 de marzo de 2014). La UCOSD como actor social. (M. d. sustentable, Entrevistador)

UCOSD. (2011). Plan Estratégico 2012-2016. Susuli: Programa de apoyo al fortalecimiento de la Unión de Campesinos Organizados de San Dionisio.

Vallaeys, F. (2004). Enseñar la ética y el desarrollo en la Universidad en la era planetaria. Lima: Maestría en gerencia social de la Pontificia Universidad Católica del Perú.

Wilber, K. (2005). Sexo, ecología y espiritualidad. Madrid: GAIA ediciones. 